\title{
Evaluation of the newly proposed simplified histological classification in Japanese cohorts of myeloperoxidase-anti- neutrophil cytoplasmic antibody-associated glomerulonephritis in comparison with other Asian and European cohorts
}

\author{
Eri Muso - Tomomi Endo - Mitsuyo Itabashi - Hiroko Kakita • Yukako Iwasaki • \\ Yu Tateishi · Toshiyuki Komiya · Toshiko Ihara • Wako Yumura • \\ Takao Sugiyama · Kensuke Joh • Kazuo Suzuki
}

Received: 22 October 2012/ Accepted: 3 December 2012/Published online: 21 December 2012

(C) The Author(s) 2012. This article is published with open access at Springerlink.com

\begin{abstract}
The prognostic value of renal biopsy in antineutrophil cytoplasmic antibody (ANCA)-associated glomerulonephritis is widely recognized; however, there is no consensus regarding its pathological classification. Berden et al. proposed a new classification of glomerulonephritis in ANCA-associated vasculitis (AAV) categorized into focal, crescentic, mixed, and sclerotic classes and showed its prognostic value in 100 international multicenter cohorts for 1- and 5-year renal outcomes. In order to evaluate whether this new classification has predictive value and reproducibility in Japanese AAV cases, 87 cohorts with
\end{abstract}

E. Muso $(\varangle) \cdot$ T. Endo · H. Kakita · Y. Iwasaki · Y. Tateishi ·

T. Komiya - T. Ihara

Division of Nephrology and Dialysis, Kitano Hospital,

The Tazuke Kofukai Medical Research Institute,

2-4-20 Ohgimachi, Kita-ku, Osaka 530-8480, Japan

e-mail: muso@kitano-hp.or.jp

M. Itabashi

4th Department of Internal Medicine,

Tokyo Women Medical College, Tokyo, Japan

W. Yumura

Preventive Medical Center, Department of Nephrology, International University of Health and Welfare Hospital,

Tochigi, Japan

T. Sugiyama

Division of Internal Medicine,

Shimoshizu National Hospital, Chiba, Japan

K. Joh

Division of Pathology, Sendai Shakaihoken Hospital,

Miyagi, Japan

K. Suzuki

Safety Control Department, University Hospital,

School of Medicine/General, Medical Education Center,

Teikyo University, Tokyo, Japan only microscopic polyangiitis in 3 limited centers in Japan were analyzed. In addition, those from Japan, Europe (Berden's cohorts) and China were compared in a recent report.

Keywords Anti-neutrophil cytoplasmic antibody . Vasculitis · Renal histology · Glomerulonephritis · Classification · Microscopic polyangiitis · Japan

\section{Introduction}

We recently proposed pathological parameters of renal lesions observed in anti-neutrophil cytoplasmic antibody (ANCA)-associated vasculitis (AAV) patients [1]. The purpose of this proposal was (1) standardization of pathological findings in AAV should be authorized in Japan; (2) comparison with the European Vasculitis Study Group (EUVAS) standardization should be available; and (3) pathological parameters correlated with specific clinical findings should be evaluated (Table 1). As a result, the pathological parameters selected were almost compatible with those selected by EUVAS except for the collapse of glomeruli as the chronicity parameter; however, further evaluation using these parameters to investigate potential markers for the probability of end-stage renal disease (ESRD) is needed.

Among the parameters listed above, the number of normal or sclerotic glomeruli was proved substantially to be a prognostic indicator of renal outcome in accordance with basal renal function [2-4]; however, no sufficient consensus exists regarding the pathological classification. Recently, using some of the glomerular parameters, an international working group of renal pathologists proposed a new histopathological classification of glomerulonephritis (GN) in 
Table 1 Pathological parameters nominated for evaluation of active and chronic lesion in ANCA-related vasculitis in Japan (comparable with EUVAS)

\begin{tabular}{ll}
\hline Glomerular lesion & \\
No. of normal glomeruli & Chronicity lesion \\
Active lesion & Sclerotic lesion \\
Mesangial proliferation & Global sclerosis \\
Endocapillary hypercellularity & Segmental sclerosis \\
Tuft necrosis & Fibrous crescent \\
Cellular, fibrocellular crescent formation & $<50 \%$ \\
$<50 \%$ & $>50 \%$ \\
$>50 \%$ & Adhesion \\
Rupture of Bowman's capsule & Collapse \\
& \\
Tubulointerstitial lesion & Chronicity lesion \\
Active lesion & Atrophic tubule \\
Tubulitis & Interstitial fibrosis \\
Disruption of tubular basement membrane & \\
Interstitial cell infiltration & \\
Granulomatous lesion & \\
Peritubular capillaritis & \\
Vascular lesions & \\
Active lesion & \\
Necrotizing & \\
Endoarteritis & \\
Cell infiltration & \\
Thromboembolism & \\
Granulomatous lesion & \\
\hline Paramicity lesion \\
\end{tabular}

${ }^{a}$ Parameter not nominated in EUVAS

AAV with four categories (focal, crescentic, mixed and sclerotic), corresponding to the severity of renal function loss in this order during a 5-year follow-up [5]. As the evaluation was performed in 100 cases, consisting of 39 cases of granulomatosis with polyangiitis (GPA) and 61 cases of microscopic polyangiitis (MPA) in 32 centers in 9 European counties, the influence of the relatively mixed races and disease types could not be excluded. In Japan, $>90 \%$ of ANCA-positive GN is diagnosed as MPA, in which renal involvement is more frequent than in GPA, as previously reported [6]. In this study, we evaluated the predictive potential of this newly proposed categorization in myeloperoxidase (MPO)-ANCA-dominant MPA patients in Japan.

\section{Patients and methods}

Eighty-seven patients with primary systemic vasculitis, in accordance with the Chapel Hill consensus criteria [7], diagnosed and treated from 2001 to 2010 in three centers (Kitano Hospital in Osaka, Tokyo Women Medical College
Table 2 Comparison among evaluations of GN histological categories with clinical background in Europe, China and Japan

\begin{tabular}{|c|c|c|c|}
\hline & $\begin{array}{l}\text { European } \\
{[5]}\end{array}$ & Japan & China [8] \\
\hline Patients (number) & 100 & 87 & 121 \\
\hline Centers (number) & 32 & 3 & 1 \\
\hline Median age (range) & $62.6(20-80)$ & $63.0(17-85)$ & $57.2(15-81)$ \\
\hline Male to female (number) & $54: 46$ & $37: 50$ & $64: 57$ \\
\hline \multicolumn{4}{|l|}{ Clinical diagnosis $(\%)$} \\
\hline GPA & $39(39)$ & 0 & $49(40.5)$ \\
\hline MPA & $61(61)$ & $87(100)$ & $68(56.2)$ \\
\hline Renal-limited vasculitis & 0 & 0 & $4(3.3)$ \\
\hline \multicolumn{4}{|c|}{ ANCA test (indirect immunofluorescence or ELISA) } \\
\hline PR3-ANCA & 45 & 0 & 13 \\
\hline MPO-ANCA & 47 & 76 & 108 \\
\hline $\operatorname{ANCA}(-)$ & 2 & 0 & 0 \\
\hline Missing & 3 & 11 & 0 \\
\hline $\begin{array}{l}\text { Median number of } \\
\text { glomeruli per biopsy } \\
\text { (range) }\end{array}$ & $14.8(10-49)$ & $26.5(10-98)$ & 25.7 (NS) \\
\hline \multicolumn{4}{|c|}{ Pathological classification number $(\%)$} \\
\hline Focal & $16(16)$ & $40(46.0)$ & $33(27.3)$ \\
\hline Crescentic & $55(55)$ & $7(8.0)$ & $53(43.8)$ \\
\hline Mixed & $16(16)$ & $26(29.9)$ & $24(19.8)$ \\
\hline Sclerotic & $13(13)$ & $14(16.1)$ & $11(9.1)$ \\
\hline \multicolumn{4}{|l|}{ Serum creatinine $(\mathrm{mg} / \mathrm{dl})$} \\
\hline Focal & NS & $1.51 \pm 1.49$ & $2.22 \pm 1.90$ \\
\hline Crescentic & & $2.42 \pm 1.67$ & $5.01 \pm 2.73$ \\
\hline Mixed & & $3.37 \pm 3.17$ & $3.86 \pm 2.69$ \\
\hline Sclerotic & & $7.52 \pm 4.92$ & $8.51 \pm 3.42$ \\
\hline Death at 1 -year follow-up & $25 / 100$ & $11 / 84$ & NS \\
\hline \multicolumn{4}{|c|}{ Renal survival at 1 -year follow-up } \\
\hline $\begin{array}{l}\text { Focal, crescentic, mixed, } \\
\text { sclerotic }(\%)\end{array}$ & $\begin{array}{l}93,84 \\
\quad 69,50\end{array}$ & $\begin{array}{r}100,86 \\
96,35\end{array}$ & $\begin{array}{r}100,73 \\
83,29\end{array}$ \\
\hline \multicolumn{4}{|c|}{ Renal survival at 5-year follow-up } \\
\hline $\begin{array}{l}\text { Focal, crescentic, mixed, } \\
\text { sclerotic }(\%)\end{array}$ & $\begin{array}{l}93,76 \\
61,50\end{array}$ & $\begin{array}{r}100,86 \\
96,29\end{array}$ & NS \\
\hline
\end{tabular}

Data of three patients were lost due to transfer to different hospitals before 1-year follow-up

$N S$ not shown in the report

in Tokyo and Shimoshizu National Hospital in Chiba) were analyzed. In all cases, renal biopsy was performed before treatment. Specimens including a minimum of 10 whole glomeruli were enrolled. Hematoxylin and eosin, methenamine silver, periodic acid-Schiff, and Masson trichrome staining were used for evaluation. The histological categorization based on glomerular lesion was performed following Berden's group [5]—focal $\geq 50 \%$ normal glomeruli, crescent $\geq 50 \%$ of glomeruli with cellular crescents, sclerotic $\geq 50 \%$ of glomeruli with global sclerosis, and mixed $<50 \%$ normal, $<50 \%$ crescentic, $<50 \%$ 
globally sclerotic glomeruli. A minimum of 6 months prognosis was observed for all patients. Renal and life survivals were analyzed at onset, 6 months, 1 year and 5 years after renal biopsy in available patients ( 87 at onset and 6 months, 84 at 1 year, 78 at 5 years).

\section{Results}

Patient profile and outcome in Japanese cohort

Median age was almost identical to the European study; however, males were dominant in Japan in contrast to a slight female dominance in Europe (Table 2).

All cases in Japan had MPA; MPO-ANCA was positive in $76 / 87(87.3 \%)$. The median glomerular number was 26.5 in Japanese samples. At 6 months follow-up, 11 patients reached ESRD and a further 8 patients had died. At 1-year follow-up, no more patients had reached ESRD and a total of 11 patients had died. At 5-year follow-up, 18 patients had died and another 12 patients had reached ESRD.

Classification of the renal biopsy in Japanese cohorts

In Japanese patients, almost half of the cases were categorized as focal $(40 / 87 ; 46.0 \%)$ with $14 / 87(16.1 \%)$ as sclerotic. Of the other 32 cases, only $7(8.0 \%)$ were categorized as crescentic, with the remaining 26 cases $(29.9 \%)$ being classed as mixed. As shown in Fig. 1, the Kaplan-Meier curve at the 5-year follow-up showed no increase of probability to ESRD in focal cases and a low increase in mixed cases; however, this increased with the ascending categories of crescentic and sclerotic GN.

Comparison among evaluations of GN histological categories in Europe, China and Japan

The predictive value and reproducibility of this new classification from Japan, Europe and China were compared in a recent report [8]. As shown in Table 2, among the 100 respective patients ( 32 centers; Europe), 121 (1; China) and 87 (3; Japan), the GPA:MPA ratio was similar between Europe and China (39:61 and 49:64) in contrast to all MPA (0:87) in Japan. On the other hand, for serum ANCA positivity, MPO-ANCA positivity was dominant in China $(89.1 \%)$ and Japan $(87.4 \%)$ compared to Europe (45\%), where there was relatively high PR3-ANCA positivity (47\%) compared with China and Japan (10.7 and $0 \%$, respectively). The average numbers of glomeruli per case were significantly higher both in Japan (26.5) and China (25.7) than in Europe (14.8). The distribution of the four histological categories of GN were similar in Europe and

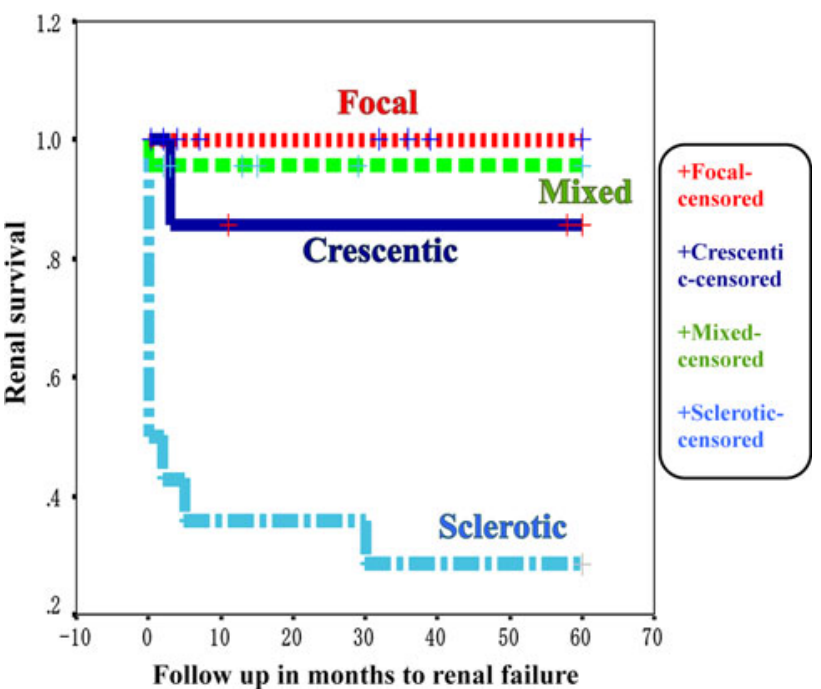

Fig. 1 Renal survival (no development of end-stage renal failure) according to the four histologic categories in Japanese cohorts

China with crescentic cases being dominant (55 and $47 \%$, respectively), whereas in Japan, the number in this category was significantly lower $(8.0 \%)$. The probability of developing ESRD increased with the ascending categories of focal, crescentic, mixed, and sclerotic in Europe, and focal, mixed, crescentic and sclerotic in China. In Japan, as mentioned above, there was no increase of probability to ESRD in focal and mixed, but there was a high increased in sclerotic, as in Europe and China.

\section{Discussion}

The histopathological findings of AAV in the kidney are considered to show a variety of lesions, of which crescentic and/or focal necrotizing GN as well as small-vessel arteritis are the most prominent [7]. In addition to the baseline laboratory data concerning renal lesions such as hematuria, proteinuria and decreased estimated glomerular filtration rate with systemic inflammatory signs such as C-reactive protein and organ involvement symptoms such as hemoptysis, renal histological findings have been expected to give highly reliable information not only to select the treatment protocol but to predict the outcome at baseline. Trials for the global standardization of active and chronic pathological parameters specifically in AAV have been performed not only in EUVAS but also in Japan, where a higher prevalence of MPA than EUVAS has been recognized, although the AAV prevalence itself is almost the same [9]. As shown in Table 1, these parameters are common findings in AAV. Almost all parameters are common in EUVAS selection, so our Japanese standardization of clinicopathologically critical parameters in AAV seems to be globally fulfilled. 
The new classification of GN into four categories (focal, crescentic, mixed, sclerotic) by selecting some of the parameters of Berden et al. [5] was highly predictive in AAV patients from multicenters in Europe. In Japan, the significantly lower frequency of crescentic and relatively higher frequency of focal cases were noted; this might be partly attributed to the earlier intervention of renal biopsy after discovering a urinary or renal function abnormality in Japan. The relatively low creatinine level of the focal group in Japan compared with that of the same group in China might support this tendency. As the progression of renal injury tends to be different between MPA and GPA, comparisons should be performed only between MPA in Europe and in Japan. This was not possible in this classification study because there were no data on the ratio of MPA in the crescentic group in Europe. In this study, the Kaplan-Meier curve revealed the highly favorable prognosis of the mixed group. This indicates that the prognosis of this group is attributed to additional pathological parameter such as tubulointerstitial or vascular lesions nominated previously in Europe and Japan. At present, at least for MPA-oriented cohorts in Japan, this classification only by glomerular parameters might be insufficient to predict the probability of progressing to ESRD.

The comparison of European, Japanese and Chinese cohorts would be highly informative. The similarity of the GPA/MPA ratio between Europe and China in contrast to that of MPO-ANCA dominancy between Japan and China indicates that many GPA are MPO-ANCA-positive in China, as Chinese authors have stated. The GPA dominancy might be attributed partly to the localization of the center at a high latitude, which has been reported to be related to the high prevalence of GPA [10]. Although the numbers in the four categories were similar between Europe and China, there was a difference in the order of the increase of probability of progressing to ESRD between mixed and crescentic. The significantly more favorable prognosis of mixed than crescentic in China is similar to Japan, where both focal and mixed rarely showed progress to ESRD.

In conclusion, the mixed group in the new classification has high heterogenicity of histological activity and chronicity, which shows the insufficiency of this classification for prediction of the probability of progressing to ESRD. Re-evaluation of the predictive value by adding other parameters such as interstitial or vascular lesions for MPAoriented cohorts is expected.

Acknowledgments This study was supported in part by a Grant-inAid for Progressive Renal Diseases Research, Research on Intractable Disease from the Ministry of Health, Labor, and Welfare of Japan.

Conflict of interest There is no conflict of interest in the preparation and submission of this manuscript.

Open Access This article is distributed under the terms of the Creative Commons Attribution License which permits any use, distribution, and reproduction in any medium, provided the original author(s) and the source are credited.

\section{References}

1. Joh K, Muso E, Shigematsu H, et al. Renal pathology of ANCArelated vasculitis: proposal for standardization of pathological diagnosis in Japan. Clin Exp Nephrol. 2008;12:277-91.

2. Bajema IM, Hagen EC, Hansen BE, et al. The renal histopathology in systemic vasculitis: an international survey study of inter- and intra-observer agreement. Nephrol Dial Transplant. 1996;11:1989-95.

3. Lind De, van Wijngaarden RA, Hauer HA, Wolterbeek R, et al. Clinical and histologic determinants of renal outcome in ANCAassociated vasculitis: a prospective analysis of 100 patients with severe renal involvement. J Am Soc Nephrol. 2006;17:2264-74.

4. Yamagata K, Usui J, Saito C, et al. ANCA-associated systemic vasculitis in Japan: clinical features and prognostic changes. Clin Exp Nephrol. 2012;16:580-8.

5. Berden AE, Ferrario F, Hagen EC, et al. Histopathologic classification of ANCA-associated glomerulonephritis. J Am Soc Nephrol. 2010;21:1628-36.

6. Fujimoto S, Uezono S, Hisanaga S, et al. Incidence of ANCAassociated primary renal vasculitis in the Miyazaki Prefecture: the first population-based, retrospective, epidemiologic survey in Japan. Clin J Am Soc Nephrol. 2006;1(5):1016-22.

7. Jennette JC, Falk RJ, Andrassy K, et al. Nomenclature of systemic vasculitides: proposal of an international consensus committee. Arthritis Rheum. 1994;37:187-92.

8. Chang DY, Wu LH, Liu G, et al. Re-evaluation of the histopathologic classification of ANCA-associated glomerulonephritis: a study of 121 patients in a single center. Nephrol Dial Transplant. 2012;27:2343-9.

9. Watts RA, Scott DG, Jayne DR, et al. Renal vasculitis in Japan and the UK-are there differences in epidemiology and clinical phenotype? Nephrol Dial Transplant. 2008;23:3928-31.

10. Watts RA, Lane SE, Scott DG, et al. Epidemiology of vasculitis in Europe. Ann Rheum Dis. 2001;60:1156-7. 